\title{
Machine-learning models to predict tacrolimus dosage in liver transplant recipients
}

\author{
Jeong-Moo Lee ${ }^{1}$, Soo Bin Yoon², Hyung-Chul Lee ${ }^{2}$, Chul-Woo Jung ${ }^{2}$, Suk Kyun Hong ${ }^{1}$, Jae-Hyung Cho ${ }^{1}$, Nam-Joon Yi ${ }^{1}$, \\ Kwang-Woong Lee ${ }^{1}$
}

${ }^{1}$ Division of Hepatobiliary, Department of Surgery, Seoul National University Hospital, Seoul, Korea
${ }^{2}$ Department of Anesthesiology, Seoul National University Hospital, Seoul, Korea

Background: Tacrolimus is the most widely used immunosuppressive agents to prevent rejection after solid organ transplantation. However, the use of tacrolimus should be cautious due to its narrow therapeutic index and variability of individual bio availabilities. Machine learning techniques could be good modality to decide optimal dosage of tacrolimus, compared with traditional statistical models, have many advantages including high power and accuracy, we have implemented a new approach to find the optimal dose of tacrolimus by machine learning technique.

Methods: We retrospectively reviewed the postoperative tacrolimus levels of patients who underwent liver transplantation at the Seoul National University Hospital from March 2016 to March 2018. We implemented an artificial intelligence model predicting future tacrolimus levels by tacrolimus concentrations in the previous two days, sex, height, and daily changing body weight. We investigated hyperparameters (the number of layers in the network and the number of nodes in each layer) using a grid search and found the model with the lowest validation error.

Results: A machine learning model was derived using data from the 187 patients. As a result of testing the model with 18 patients, the predicted value of the model had an error of $1.5 \mathrm{ug} / \mathrm{L}$ from the actual measured tacrolimus level. Simulating the model in the random case with a calculated tacrolimus dose to ensure the next drug concentration to be within the therapeutic range, more than $95 \%$ of the final predicted tacrolimus level comes in the therapeutic window.

Conclusions: This is the first study to use machine learning models to predict optimal dosage after liver transplantation. The machine-learning model is useful to decide the optimal dose of tacrolimus immediate postoperative period after liver transplantation.

Corresponding author: Jeong-Moo Lee

E-mail:jmleetpl@gmail.com

(c) The Korean Society for Transplantation

This is an Open Access article distributed under the terms of the Creative Commons Attribution Non-Commercial License (http://creativecommons.org/licenses/by-nc/4.0/) which permits unrestricted non-commercial use, distribution, and reproduction in any medium, provided the original work is properly cited. 